\title{
BMJ Open Associations of perceived interparental relationship, family harmony and family happiness with smoking intention in never-smoking Chinese children and adolescents: a cross- sectional study
}

Tzu Tsun Luk, ${ }^{1}$ Man Ping Wang, ${ }^{1}$ Lok Tung Leung, ${ }^{2}$ Yongda Wu, ${ }^{1}$ Jianjiu Chen, ${ }^{2}$ Tai Hing Lam, ${ }^{2}$ Sai Yin $\mathrm{Ho}^{2}$

To cite: Luk TT, Wang MP, Leung LT, et al. Associations of perceived interparental relationship, family harmony and family happiness with smoking intention in neversmoking Chinese children and adolescents: a crosssectional study. BMJ Open 2017;7:e017523. doi:10.1136/ bmjopen-2017-017523

- Prepublication history for this paper is available online. To view these files please visit the journal online (http://dx.doi. org/10.1136/bmjopen-2017017523).

Received 27 April 2017 Revised 25 August 2017 Accepted 31 August 2017

CrossMark

${ }^{1}$ School of Nursing, The University of Hong Kong, Hong Kong, China

${ }^{2}$ School of Public Health, The University of Hong Kong, Hong kong, China

Correspondence to Dr Man Ping Wang; mpwang@hku.hk

\section{ABSTRACT}

Objective To examine the associations of perceived interparental relationship, family harmony and family happiness with smoking intention in never-smoking Chinese children and adolescents in Hong Kong.

Design, settings and participants Cross-sectional surveys of 15753 primary (grades 4-6) and 38398 secondary (grades 7-12) never-smoking students from 71 to 75 randomly selected primary and secondary schools in Hong Kong, 2012-2013.

Measurements Outcome variable was smoking intention which denoted any affirmative response to smoke within the coming year or when a cigarette was offered by a good friend. Exposure variables were perceived interparental relationship and family harmony each measured on a fivepoint scale from 'very good' to 'very bad' and perceived family happiness on a four-point scale from 'very happy' to 'not happy at all'. Potential confounders included age, sex, family structure, perceived family affluence, parental smoking and sibling smoking.

Results In primary students, the adjusted ORs (AORs) (95\% $\mathrm{Cl}$ ) of smoking intention generally increased with more negative perception of the family relationship: up to 3.67 (1.91 to 7.05 ) for interparental relationship, 7.71 (4.38 to 13.6) for family harmony and 5.40 (3.41 to 8.55) for family happiness. For secondary students, the corresponding AORs $(95 \% \mathrm{Cl})$ were 2.15 (1.64 to 2.82) for interparental relationship, 2.98 (2.31 to 3.84 ) for family harmony and 2.61 (1.80 to 3.79) for family happiness. All $\mathrm{p}$ for trend $<0.001$.

Conclusions More negatively perceived interparental relationship, family harmony and family happiness were associated with higher odds of smoking intention with doseresponse relationships in never-smoking Chinese children and adolescents in Hong Kong. Children's perception of their family relationship may be an important intervening point for preventing youth from initiating smoking.

\section{INTRODUCTION}

Youth smoking has remained a public health challenge worldwide including Hong Kong, a
Strengths and limitations of this study

- This study is the first to show strong, dose-response association of family relationship with smoking intention in Chinese children and adolescents, an understudied population.

- Data were collected from two large and representative samples of Hong Kong children and adolescents with high response rate.

- Cross-sectional study design precludes causal inference.

Self-reported measures were used.

city of China with a low daily smoking prevalence of $10.5 \% .^{1}$ Recent figures have shown an upward trend in ever smoking rate among local primary students. ${ }^{1}$ The age of smoking initiation is also declining in other regions in China. $^{2-4}$ Early smoking predicts long-term tobacco use, ${ }^{5}$ more difficult quitting ${ }^{6}$ and higher risk of all-cause mortality in adulthood. ${ }^{7}$ Identifying risk factors of smoking initiation in Chinese youth has major local and global health implications.

The family has important influence on youth smoking. Lower family socioeconomic status and having smoking family members predict while parental monitoring and authoritative parenting protect against youth smoking initiation. ${ }^{8-10}$ Other family-related risk factors include non-intact family structure, low parental education level and parental approval of smoking. ${ }^{9}$ Studies in Hong Kong also show that parental smoking, exposure to secondhand smoke at home and overestimation of peer smoking prevalence were prospectively linked to youth smoking initiation. $^{1112}$ 
Despite the importance of family relationship in Chinese culture, little is known about its role in youth smoking initiation. Under collectivism, family harmony is deemed the foundation of family functioning in the Chinese culture. ${ }^{13}$ It is the ideal state of family relationship emphasising the sense of togetherness and absence of intrafamilial conflict through mutual respect and communication. ${ }^{13} 14$ Family happiness is the outcome of a harmonious family in which members care and support each other and feel emotionally secured. ${ }^{13}$ Conflict between family members is a major threat to family harmony, which in turn compromises family happiness. ${ }^{13} 14$ Children who have witnessed conflicts between their parents and perceived family as conflictual and unhappy have greater risk of emotional distress, depression and maladjustment, ${ }^{15-17}$ all of which have been shown to predict youth smoking initiation in Westerners ${ }^{18} 19$ and Chinese. ${ }^{20}$ Many studies have found youth smoking initiation associated with negative parentchild relationship, ${ }^{21-24}$ but evidence on its association with interparental relationship is lacking. Never has the role of family happiness in youth smoking initiation been reported. A cross-sectional study investigated how family disharmony was associated with ever smoking in Chinese, but the study was small $(\mathrm{n}=182)$ and included adolescents mostly aged $13 .{ }^{25}$

Family harmony has remained a core value and an index of societal stability in Hong Kong. ${ }^{26}$ Studying the determinants of youth smoking in Hong Kong helps develop culturally appropriate smoking prevention programmes that may also benefit mainland China and elsewhere. ${ }^{27}$ The theory of planned behaviour postulates that behavioural intention precedes the actual behaviour, ${ }^{28}$ as have been observed in youths intended to initiate smoking. ${ }^{9}{ }^{29}$ We therefore tested the associations of perceived interparental relationship, family harmony and family happiness with smoking intention in two large, population-representative, cross-sectional samples of never-smoking Chinese children and adolescents in Hong Kong.

\section{METHODS}

\section{Study design}

We analysed cross-sectional data from the School-based Survey on Smoking among Students 2012/2013 collected during October 2012 to April 2013 in Hong Kong. Detail of the study design has been reported elsewhere.$^{30}$ Briefly, schools were randomly selected from all 18 districts in Hong Kong in proportion to the total number of schools in the respective districts. All grades 4-6 primary students and grades 7-12 secondary students in the selected schools were selected. Invitation letters were sent to their parents via the students for passive consents, where declining parents asked the student to return an empty questionnaire. Students voluntarily completed an anonymous questionnaire in Chinese, which was immediately collected and sealed in an opaque envelope by research personnel in front of the students. Core questions in the survey were adapted from Global Youth Tobacco Survey. Ethical approval was granted by the Institutional Review Board of the University of Hong Kong/Hospital Authority Hong Kong West Cluster. A total of 16316 students from 71 primary schools and 45857 students from 75 secondary schools completed the survey. Response rates were about $96 \%$ at the student level and $20 \%$ at the school level. School refusals were largely due to administrative issues (eg, busy class schedule). We randomly sampled schools with similar funding source, teaching medium and sex composition in the same district for replacement of the rejected school.

\section{Main measures}

\section{Outcome measures}

Similar to previous studies, we measured smoking intention with two items: 'smoke in the next 12 months' and 'smoke if one of your good friends offers you a cigarette' each with four-point response options of 'definitely not', 'probably not', 'probably will' and 'definitely will'. ${ }^{29} 31$ Students who reported 'definitely not' for both questions were classified as having no intention to smoke and otherwise as having an intention to smoke.

\section{Exposure measures}

As the first population study on family relationship and smoking in Hong Kong, we developed three questions based on our findings from two local qualitative studies on family well-being. ${ }^{1314}$ One question assessed perceived interparental relationship: "What do you think is the relationship between your father and mother?" with five-point responses of 'very good', 'good', 'fair', 'bad' and 'very bad' and 'not applicable'. Family harmony was measured with students rating the item 'My family gets along well' on a five-point scale of 'strongly agree', 'agree', 'fair', 'disagree' and 'strongly disagree', ${ }^{32}$ which were renamed as 'very good' to 'very bad' for presentation. Perceived family happiness was assessed by asking: "All things considered, you think your family is:" with response options of 'very happy', 'happy', 'not very happy' and 'not happy at all'. These three items had satisfactory internal consistency (Cronbach's alpha $=0.78$ in primary and 0.70 in secondary students) in our samples and 8-day test-retest reliability in a separate sample of 329 primary students (mean age 10.7; boys $51.9 \%$ ) with intraclass correlation coefficient of 0.85 for interparental relationship, 0.67 for family harmony and 0.73 for family happiness.

We created a composite variable namely number of poor family relationship factors (range $0-3$ ) by summing the perceived interparental relationship, family harmony (each dichotomised into 0 for 'very good/good' and 1 for 'fair/bad/very bad') and family happiness ( 0 for 'very happy/happy' and 1 for 'not very happy/nothappy at all'). The three items (ie, $0=$ 'very good' to $4=$ 'very bad') were also summed to give a poor family relationship score which ranged from 0 to 11 . Although children's perceptions of family relationship may differ from those of other family members, ${ }^{33}$ it should be their subjective assessment 


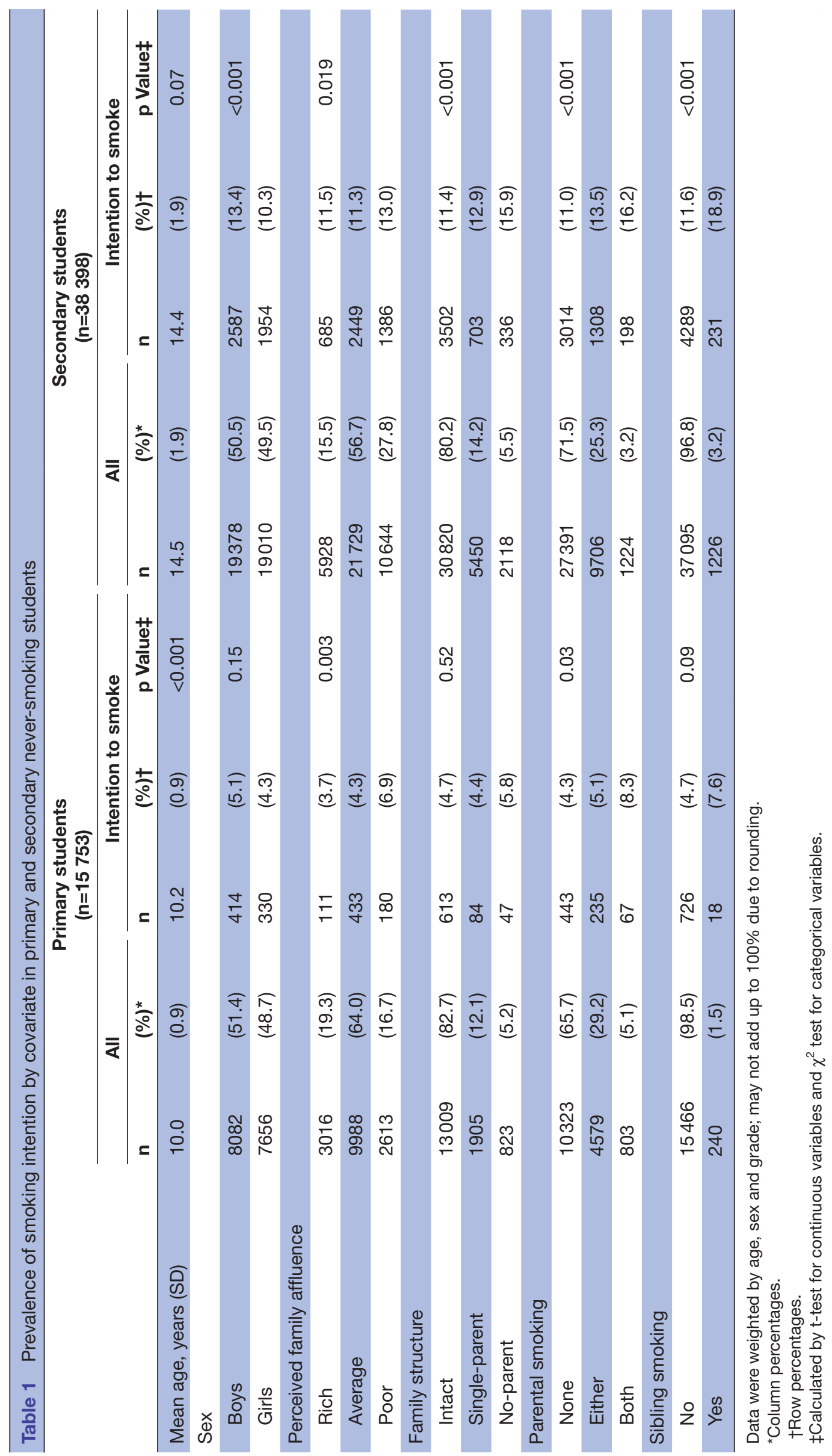


Table 2 Associations of perceived interparental relationship, family harmony, family happiness and family relationship with smoking intention in primary students

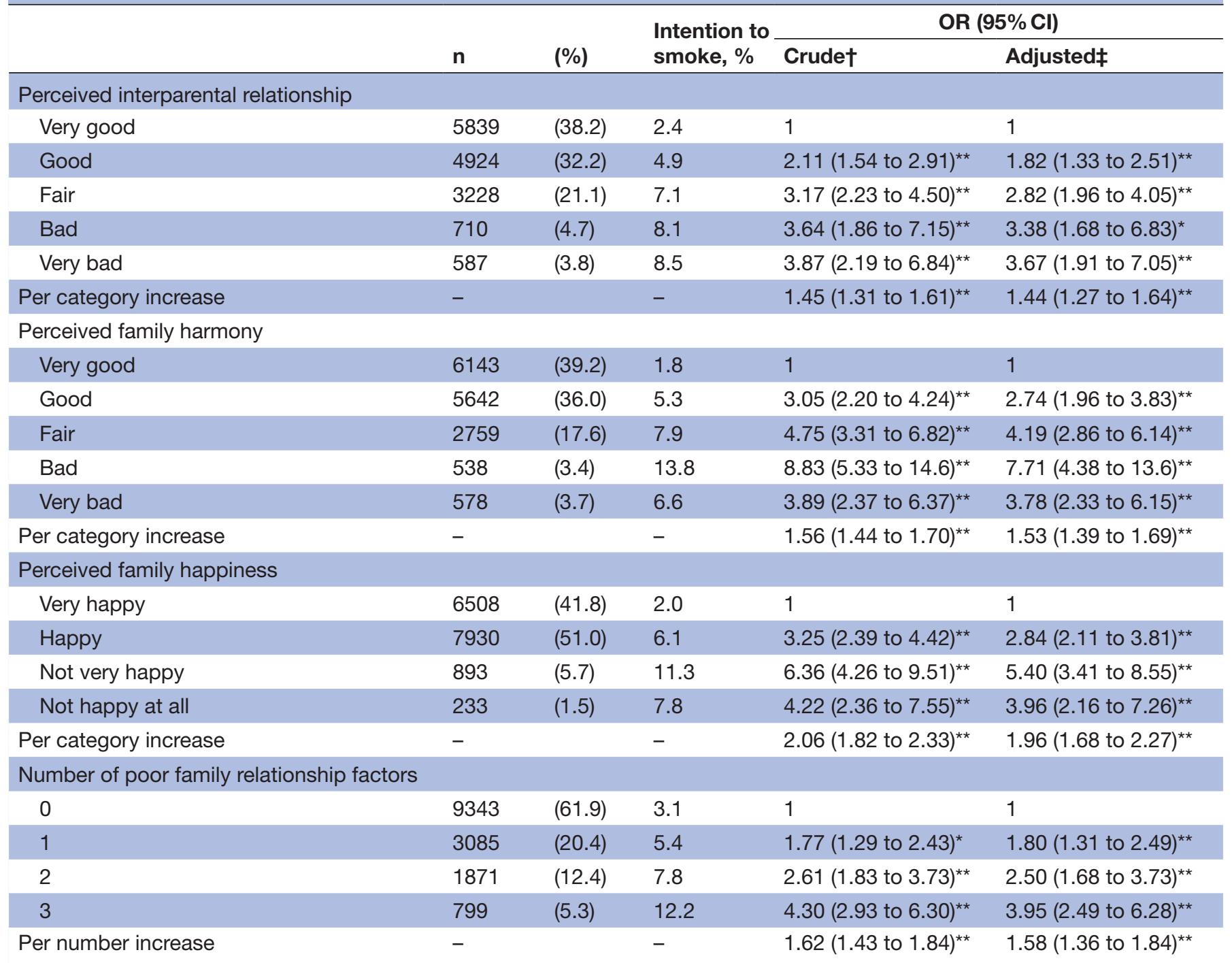

Data were weighted by age, sex and grade; may not add up to $100 \%$ due to rounding.

†Adjusted for school clustering effect only.

$\ddagger$ Adjusted additionally for age, sex, perceived family affluence, family structure, parental smoking and sibling smoking.

${ }^{*} \mathrm{p}<0.01$.

${ }^{* *} \mathrm{p}<0.001$

that would be more meaningful and relevant to the development of their smoking intentions.

\section{Potential confounders}

We also collected data on age, sex and perceived family affluence. The advantage and reliability of perceived family affluence as a proxy measure of family socioeconomic status for youths in Hong Kong has been reported previously. ${ }^{34}$ We also assessed family structure by asking if the students were living with their mothers and fathers, with responses classified into intact (living with both parents), single-parent (either mother or father) and no-parent (neither). ${ }^{35}$ Household smoking were determined by asking: "Among those you live with, who is/are smoker(s)?" with response options of 'father', 'mother', 'brother(s)' and 'sister(s)' Parental smoking was classified into none, either (father or mother) and both (father and mother). Sibling smoking denoted having either brother(s) or sister(s), or both as smokers.

\section{Statistical analysis}

All analyses were performed in Stata/ICV.13.1 with svy commands to account for intraclass correlation (school clustering effect) due to the study design. Data were weighted by age, sex and grade distribution of the official Hong Kong 2012/2013 student enrolment statistics. To avoid reverse causality, only never-smokers were analysed. These included $15753(97.5 \%)$ primary students and $38398(85.0 \%)$ secondary students. $\mathrm{X}^{2}$ tests and t-tests were used to compare students with or without smoking intention by covariates. Multivariable logistic regression computed adjusted ORs (AORs) and 95\% CI 
Adjusted log(odds ratios) of smoking intention

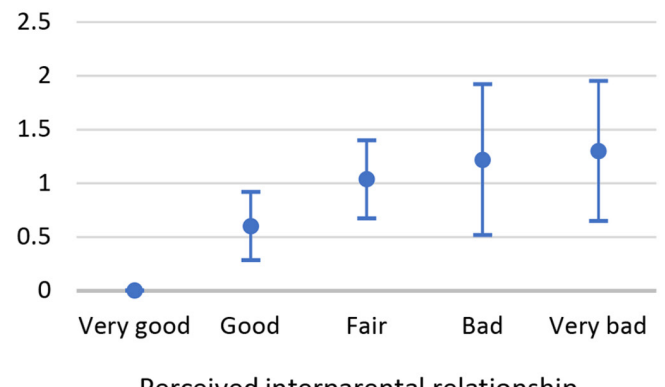

Perceived interparental relationship

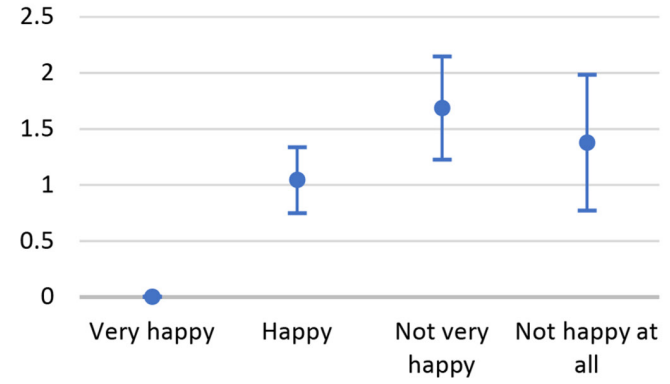

Perceived family happiness

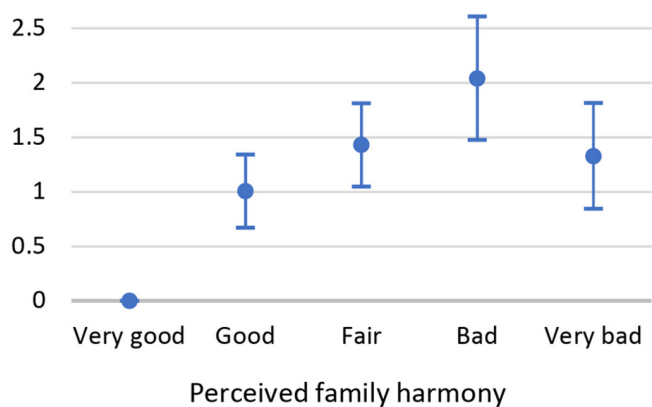

2.5

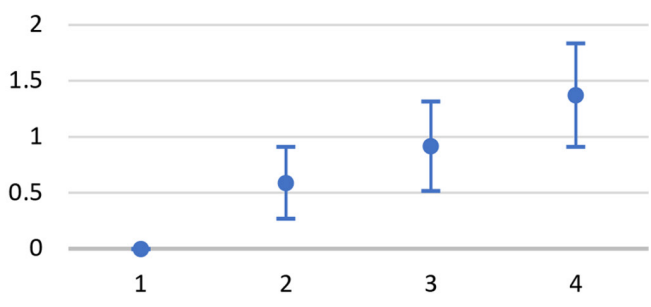

Number of poor family relationship factors

Figure 1 Adjusted ORs and $95 \% \mathrm{Cl}$ (log-scale) of smoking intention by categories of perceived interparental relationship, family harmony, family happiness, and family relationship in primary students.

of smoking intention in relation to perceived interparental relationship, family harmony and family happiness (the three family factors) and the two composite poor family relationships using complete-case analysis. As having smoking household members may contribute to perceived family unhappiness in children ${ }^{36}$ and family disharmony, ${ }^{37}$ all regression analyses were adjusted for parental smoking and sibling smoking in addition to age, sex and perceived family affluence and family structure. The F-adjusted mean residual goodness-of-fit tests verified that all adjusted logistic regression models have satisfactory fit $(\mathrm{p}=0.10$ to 0.82$){ }^{38}$ The three family factors were not included in the same model because moderate to relatively strong correlations among the three family factors were observed in both primary (Spearman's correlation coefficients ranged from 0.56 to 0.62 ) and secondary samples $(0.47$ to 0.65$)$. All predictor variables were also analysed as continuous variables to test their linear relationships with smoking intention ( $p$ for trend). A two-sided $p<0.05$ denotes statistical significance.

\section{RESULTS}

\section{Primary students}

The prevalence of smoking intention in all primary neversmokers was $4.7 \%$ (95\% CI $4.1 \%$ to $5.5 \%$ ). Table 1 shows the sample characteristics. Students with smoking intention were significantly older than those without smoking intention (10.2 vs $9.9 ; \mathrm{p}<0.001)$ and were associated with perceived poorer family affluence $(\mathrm{p}=0.003)$ and higher number of smoking parents $(p=0.03)$ but not with sex $(\mathrm{p}=0.15)$, intactness of family structure $(\mathrm{p}=0.52)$ or sibling smoking $(\mathrm{p}=0.09)$.

The prevalence of smoking intention generally increased with more negative responses in perceived interparental relationship, family harmony and family happiness (the three family factors), and poor family relationship (table 2 and figure 1). After adjusting for age, sex, perceived family affluence, family structure, parental smoking and sibling smoking, strong graded associations between each of the three family factors and smoking intention were observed (all $p$ for trend $<0.001$ ). Higher odds of smoking intention were observed in primary students with 'good' to 'very bad' perceived interparental relationship (AORs ranging from 1.82 to 3.67 ) and family harmony (AORs ranging from 2.74 to 7.71 ) relative to those reporting 'very good' interparental relationship and family harmony. The corresponding AORs ranged from 2.84 to 5.40 for students perceiving family happiness as 'happy' to 'not happy at all' (vs 'very happy'). Significant graded association between poor family relationship and smoking intention was observed ( $\mathrm{p}$ for trend $<0.001$ ) with AORs (95\% CI) increasing from 1.80 (1.31 to 2.49) for one factor to 3.95 (2.49 to 6.28) for three factors (all $\mathrm{p}<0.01)$. Each unit increase in poor family relationship score (0-11) was associated with 26\% (95\% CI $19 \%$ to $33 \%$ ) increased odds of smoking intention.

\section{Secondary students}

For never-smoking secondary students, $11.8 \%$ (95\% CI $11.1 \%$ to $12.6 \%$ ) reported an intention to smoke, which was associated with male sex $(\mathrm{p}<0.001)$, poor perceived 
Table 3 Associations of perceived interparental relationship, family harmony, family happiness and family relationship with smoking intention in secondary students

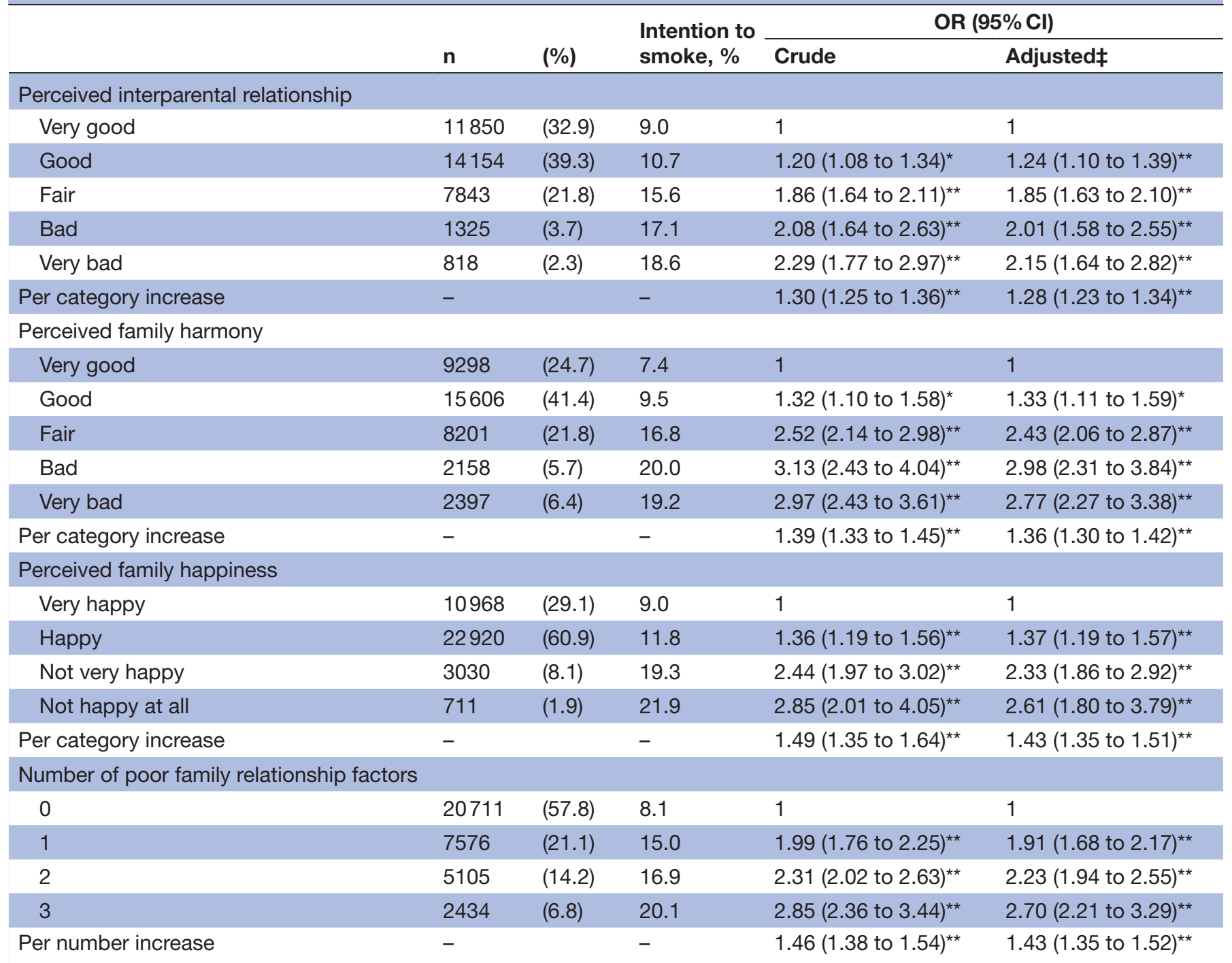

Data were weighted by age, sex and grade; may not add up to $100 \%$ due to rounding.

†Adjusted for school clustering effect only.

$\ddagger$ Adjusted additionally for age, sex, perceived family affluence, family structure, parental smoking and sibling smoking.

${ }^{*} \mathrm{p}<0.01$.

${ }^{* *} \mathrm{p}<0.001$.

family affluence $(\mathrm{p}=0.019)$, non-intact family structure $(\mathrm{p}<0.001)$, having more smoking parents $(\mathrm{p}<0.001)$ and sibling smoking $(\mathrm{p}<0.001)$ (table 1$)$. Mean age was similar in students with (14.4) or without (14.5) smoking intention $(\mathrm{p}=0.07)$.

Overall, the prevalence of smoking intention increased with more negative responses in interparental relationship, family harmony and family happiness, and poor family relationship (table 3 and figure 2). We found significant dose-response relationship between the three family factors and smoking intention (all $\mathrm{p}$ for trend $<0.001$ ), after adjusting for sociodemographic factors, parental smoking and sibling smoking. The AORs ranged from 1.24 to 2.15 for more negative perception of interparental relationship, 1.33 to 2.98 for family harmony and 1.37 to
2.61 for family happiness (all $\mathrm{p}<0.01$ ). The AORs of smoking intention increased with poor family relationship factors with significant graded relationship ( $\mathrm{p}$ for trend $<0.001)$, from 1.91 (1.68 to 2.17) for one factor to 2.70 (2.21 to 3.29) for three factors (all $\mathrm{p}<0.001$ ). Each unit increase in poor family relationship score (0-11) was associated with $19 \%$ (95\% CI $16 \%$ to $22 \%$ ) increased odds of smoking intention.

To test the stability of the estimates, we further add number of smoking peers (none/some/half + ), attitude towards smoking (negative/neutral/positive) and knowledge in smoking harm (good/poor) in the multivariable models. Additional adjustment for these variables did not change the estimates substantially in both primary and secondary students (data not shown). 
Adjusted log(odds ratios) of smoking intention

1.5

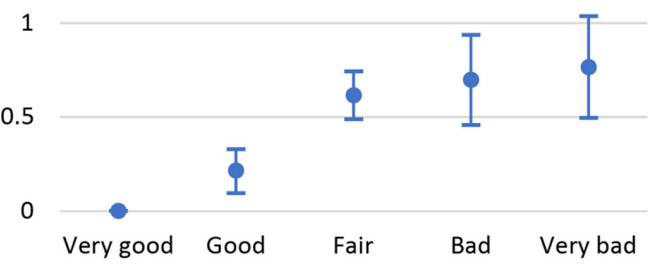

Perceived interparental relationship

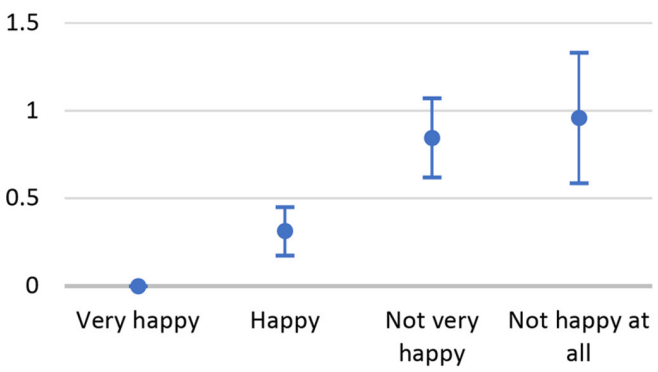

Perceived family happiness

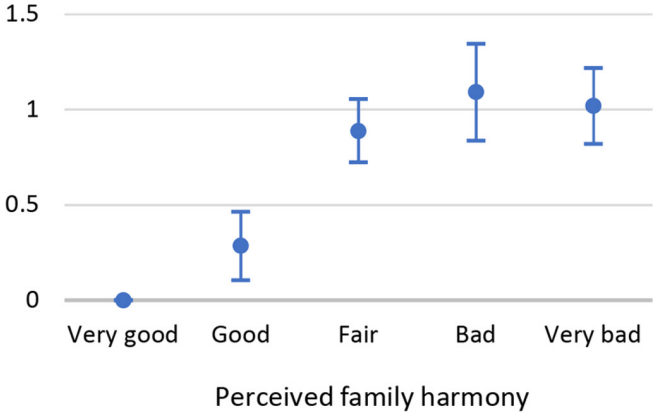

1.5

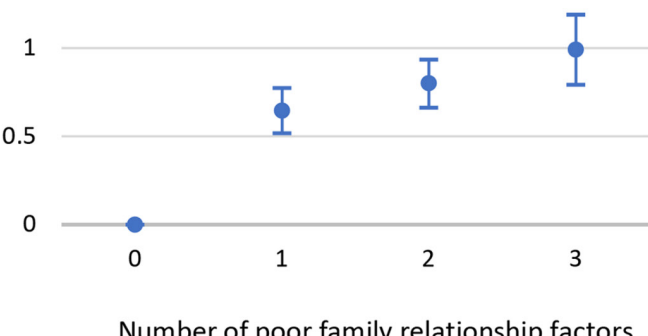

Number of poor family relationship factors

Figure 2 Adjusted ORs and 95\% Cl (log-scale) of smoking intention by categories of perceived interparental relationship, family harmony, family happiness, and family relationship in secondary students.

\section{DISCUSSION}

This study found strong, significant associations of interparental relationship, family harmony and family happiness with smoking intention in both never-smoking Chinese children and adolescents with dose-response relationships. The result for family harmony is consistent with that of the small cross-sectional study on family disharmony and ever smoking in Chinese adolescents in Wuhan, ${ }^{25}$ and studies in other cultures that generally show family conflict is predictive of youth smoking initiation. ${ }^{21-2439}$ Our study contributes to previous research by showing that the association may be dose dependent and extending the understanding of interparental relationship, an understudied family factor, as a significant determinant of youth smoking initiation. The ORs were very similar with or without adjustment of potential confounders, suggesting that the observed associations were unlikely attributable to confounding effects and that family relationship may independently predict smoking intention in Chinese never-smoking youths. The robust findings on different indicators of family relationships suggest their significant influences on smoking intention in Chinese children and adolescents.

Our findings were based on Chinese never-smoking children and adolescents. As risks of future smoking in relation to psychosocial predictors are higher in ever smokers than never-smokers, ${ }^{40}$ the association between family relationship and smoking intention may be stronger in students who have experienced cigarettes. The social significance of family may render family relationship a more influential determinant of smoking intention in youths from China and other similar cultures in Asia than their Western counterparts. Nevertheless, given the high incidence of divorce (which reflects poor interparental relationship) in Western nations with USA having the highest rate and Europe's on an increasing trend, ${ }^{41}$ testing the role of interparental relationship on smoking initiation in Western youth population is warranted.

Poor family relationship may lead to smoking in children and adolescents through different pathways. As described earlier, psychosocial stressors may mediate children's poor perceptions of family environment and smoking initiation. However, impaired parenting and parent-child relationship may also be important mediators. Conflict between parents can disrupt parenting routines, ${ }^{42}$ which may render parental monitoring, parental disapproval of smoking and other parenting practices ineffective against youth smoking initiation. The negative emotion of parents in hostile relation may also transfer or 'spillover' to children and compromise parent-child relationship. ${ }^{43}$ Weak bonding with the family in turn predisposes the children to attachment with deviant peers who may promote smoking. ${ }^{44}$

Empirical evidence from neurocognitive research supports the role of poor family relationship in adolescent smoking. Social experience can modulate the development of two neural systems in adolescents: ${ }^{45} 46$ the cognitive control system, which regulates behaviour but remains immature throughout adolescence, and the reward processing system, which has heightened sensitivity during adolescence and underlies youths' increased temptation to try new, potentially pleasurable yet risky activity such as smoking. ${ }^{47}$ A functional neuroimaging study showed that positive family relationship might 
improve adolescents' cognitive and decision-making abilities to regulate their behaviours, which were linked to reduced tendency to take risk. ${ }^{48}$ In contrast, adolescents from families with more conflicts and poorer cohesion had longitudinal impairment in cognitive control and increased risk-taking propensity. ${ }^{49}$

Parents should be warned that conflict among themselves or family disharmony and unhappiness may contribute to smoking ideation in children. They should be encouraged to seek appropriate help (eg, family counselling services) to manage unresolved family conflicts. Our findings also suggest that children's perceptions of their family relationship may be an important intervening point for preventing youth from initiating smoking.

This study has several limitations. First, the cross-sectional design of the survey precludes causal inference of interparental and family relationship on children and adolescent smoking intention. Prospective studies are warranted to confirm the results. However, it is unlikely that youth smoking intention, which is not easily observable in the family, could affect interparental relationship. Second, although confidentiality is reassured to encourage candid reporting, reporting (information) bias remained a distinct possibility. The sensitive nature of the questions could lead to random under-reporting, which might bias the associations towards the null. Third, the low school-response rate might contribute to non-response bias. However, schools' rejections were largely due to administrative issues, which was not related to student smoking. Furthermore, based on the Hong Kong 2012/2013 student enrolment statistics, our student samples were comparable to their underlying population in sex (Cohen's effect size 0.04 in primary and 0.05 in secondary students), age $(0.62 ; 0.18)$ and grade $(0.01$; $0.21) .^{50}$ The moderate effect size observed for age in primary students was attributed to the difference in reference time frame between the official statistic (mid-September 2012) and data collection (October 2012-April 2013) as students reported their age at the time of completing the survey.

\section{CONCLUSIONS}

Perceived lower levels of interparental relationship, family harmony and family happiness were associated with increased odds of smoking intention with doseresponse relationships in Chinese children and adolescents. Prospective studies are warranted to confirm the findings.

Acknowledgements We thank the schools and students for their participation in the survey.

Contributors MPW, SYH and THL designed the study. MPW, LTL, JC and SYH collected the data. TTL, MPW, YW and JC analysed the data. TTL and MPW drafted the manuscript. All authors critically revised and approved the final version of the manuscript.

Funding The work was supported by the Food and Health Bureau, Government of the Hong Kong SAR.

Competing interests None declared.

\section{Patient consent Obtained.}

Ethics approval Institutional Review Board of the University of Hong Kong/Hospital Authority Hong Kong West Cluster.

Provenance and peer review Not commissioned; externally peer reviewed.

Data sharing statement № additional data are available.

Open Access This is an Open Access article distributed in accordance with the Creative Commons Attribution Non Commercial (CC BY-NC 4.0) license, which permits others to distribute, remix, adapt, build upon this work non-commercially, and license their derivative works on different terms, provided the original work is properly cited and the use is non-commercial. See: http://creativecommons.org/ licenses/by-nc/4.0/

(C) Article author(s) (or their employer(s) unless otherwise stated in the text of the article) 2017. All rights reserved. No commercial use is permitted unless otherwise expressly granted.

\section{REFERENCES}

1. Census and Statistics Department of Hong Kong Special Administrative Region. Thematic house holdsurvey report no.59. 2016. http://www.digital21.gov.hk/eng/statistics/download/ householdreport2016_59.pdf

2. Qian J, Cai M, Gao J, et al. Trends in smoking and quitting in China from 1993 to 2003: National Health Service Survey data. Bull World Health Organ 2010;88:769-76.

3. Zhang J, Ou JX, Bai CX. Tobacco smoking in China: prevalence, disease burden, challenges and future strategies. Respirology 2011;16:1165-72.

4. Li S, Meng L, Chiolero A, et al. Trends in smoking prevalence and attributable mortality in China, 1991-2011. Prev Med 2016;93:82-7.

5. Chassin L, Presson CC, Sherman SJ, et al. The natural history of cigarette smoking: predicting young-adult smoking outcomes from adolescent smoking patterns. Health Psychol 1990;9:701-16.

6. Chen J, Millar WJ. Age of smoking initiation: implications for quitting. Health Rep 1998;9:39-46.

7. Chen Z, Peto R, Zhou M, et al. Contrasting male and female trends in tobacco-attributed mortality in China: evidence from successive nationwide prospective cohort studies. Lancet 2015;386:1447-56.

8. Leonardi-Bee J, Jere ML, Britton J. Exposure to parental and sibling smoking and the risk of smoking uptake in childhood and adolescence: a systematic review and meta-analysis. Thorax 2011;66:847-55.

9. Wellman RJ, Dugas EN, Dutczak H, et al. Predictors of the onset of cigarette smoking: a systematic review of longitudinal populationbasedstudies in youth. Am J Prev Med 2016;51:767-78.

10. Thomas RE, Baker PR, Thomas BC, et al. Family-based programmes for preventing smoking by children and adolescents. Cochrane Database Syst Rev 2015:CD004493.

11. Wang MP, Ho SY, Lam TH. Parental smoking, exposure to secondhand smoke at home, and smoking initiation among young children. Nicotine Tob Res 2011;13:827-32.

12. Wang MP, Ho SY, Lo WS, et al. Overestimation of peer smoking prevalence predicts smoking initiation among primary school students in Hong Kong. J Adolesc Health 2011;48:418-20.

13. Lam WW, Fielding R, McDowell I, et al. Perspectives on family health, happiness and harmony $(3 \mathrm{H})$ among Hong Kong Chinese people: a qualitative study. Health Educ Res 2012;27:767-79.

14. Chan SS, Viswanath K, Au DW, et al. Hong Kong Chinese community leaders' perspectives on family health, happiness and harmony: a qualitative study. Health Educ Res 2011;26:664-74.

15. Cummings EM, Davies PT. Effects of marital conflict on children: recent advances and emerging themes in process-oriented research. J Child Psychol Psychiatry 2002;43:31-63.

16. Raschke HJ, structure F. Family structure, family happiness, and their effect on college students' personaland social adjustment. Fam Court Rev 1977;15:30-3.

17. Rhoades KA. Children's responses to interparental conflict: a meta-analysis of their associations with child adjustment. Child Dev 2008;79:1942-56.

18. Koval JJ, Pederson LL, Mills CA, et al. Models of the relationship of stress, depression, and other psychosocial factors to smoking behavior: a comparison of a cohort of students in grades 6 and 8 . Prev Med 2000;30:463-77.

19. O'Loughlin J, Karp I, Koulis T, et al. Determinants of first puff and daily cigarette smoking in adolescents. Am J Epidemiol 2009;170:585-97. 
20. Lam TH, Stewart SM, Ho SY, et al. Depressive symptoms and smoking among Hong Kong Chinese adolescents. Addiction 2005;100:1003-11.

21. Fleming $\mathrm{CB}, \mathrm{Kim} \mathrm{H}$, Harachi TW, et al. Family processes for children in early elementary school as predictors of smoking initiation. $J$ Adolesc Health 2002;30:184-9.

22. Brook JS, Zhang C, Finch SJ, et al. Adolescent pathways to adult smoking: ethnic identity, peer substance use, and antisocial behavior. Am J Addict 2010;19:178-86.

23. Mahabee-Gittens EM, Xiao Y, Gordon JS, et al. Continued importance of family factors in youth smoking behavior. Nicotine Tob Res 2012;14:1458-66.

24. White J. The contribution of parent-child interactions to smoking experimentation in adolescence: implications for prevention. Health Educ Res 2012;27:46-56.

25. Trinidad DR, Chou CP, Unger JB, et al. Family harmony as a protective factor against adolescent tobacco and alcohol use in Wuhan, China. Subst Use Misuse 2003;38:1159-71.

26. Ho SSM, Chan RS. Social harmony in Hong Kong: level, determinants and policy implications. Soc Indic Res 2009;91:37-58.

27. Koplan JP, An WK, Lam RM. Hong Kong: a model of successful tobacco control in China. Lancet 2010;375:1330-1.

28. Ajzen I, Madden TJ. Prediction of goal-directed behavior: Attitudes, intentions, and perceived behavioral control. J Exp Soc Psychol 1986;22:453-74.

29. Pierce JP, Choi WS, Gilpin EA, et al. Validation of susceptibility as a predictor of which adolescents take up smoking in the United States. Health Psychol 1996;15:355-61.

30. Wang MP, Ho SY, Leung LT, et al. Electronic cigarette use and its association with smoking in Hong Kong Chinese adolescents. Addict Behav 2015;50:124-7.

31. Sterling KL, Ford KH, Park H, et al. Scales of smoking-related selfefficacy, beliefs, and intention: assessing measurement invariance among intermittent and daily high school smokers. Am J Health Promot 2014;28:310-5.

32. Fabrizio CS, Lam TH, Hirschmann MR, et al. Parental emotional management benefits family relationships: A randomized controlled trial in Hong Kong, China. Behav Res Ther 2015;71:115-24.

33. Noller P, Callan VJ. Adolescent and parent perceptions of family cohesion and adaptability. J Adolesc 1986;9:97-106.

34. Ho SY, Wong BY, Lo WS, et al. Neighbourhood food environment and dietary intakes in adolescents: sex and perceived family affluence as moderators. Int J Pediatr Obes 2010;5:420-7.
35. Mak KK, Ho SY, Thomas GN, et al. Family structure, parent-child conversation time and substance use among Chinese adolescents. BMC Public Health 2010;10:503.

36. Chen JJ, Ho SY, Au WM, et al. Family Smoking, Exposure to Secondhand Smoke at Home and Family Unhappiness in Children. Int J Environ Res Public Health 2015;12:14557-70.

37. Wang $X$, Wang MP, Viswanath $\mathrm{K}$, et al. Smoking and secondhand smoke exposure at home were associated with poor perceived family well-being: findings of family project. PLoS One 2016;11:e0161761.

38. Archer KJ, Lemeshow S. Goodness-of-fit test for a logistic regression model fitted using survey sample data. Stata J 2006;6:97-105.

39. Rajesh V, Diamond PM, Spitz MR, et al. Smoking initiation among Mexican heritage youth and the roles of family cohesion and conflict. $J$ Adolesc Health 2015;57:24-30.

40. Kremers SP, Mudde AN, de Vries H. "Kicking the initiation": do adolescent ex-smokers differ from other groups within the initiation continuum? Prev Med 2001;33:392-401.

41. Amato PR, James S. Divorce in Europe and the United States: commonalities and differences across nations. Fam Sci 2010;1:2-13.

42. Krishnakumar A, Buehler C. Interparental conflict and parenting behaviors: a meta-analytic review. Fam Relat 2000;49:25-44.

43. Erel $O$, Burman $B$. Interrelatedness of marital relations and parent-child relations: a meta-analytic review. Psychol Bull 1995;118:108-32.

44. Elliott DS, Ageton SS, Huizinga D. Explaining delinquency and drug use. Beverly Hills, CA: Sage, 1985.

45. Blakemore SJ, Mills KL. Is adolescence a sensitive period for sociocultural processing? Annu Rev Psychol 2014;65:187-207.

46. Schriber RA, Guyer AE. Adolescent neurobiological susceptibility to social context. Dev Cogn Neurosci 2016;19:1-18.

47. Geier CF. Adolescent cognitive control and reward processing: implications for risk taking and substance use. Horm Behav 2013;64:333-42.

48. Telzer EH, Fuligni AJ, Lieberman MD, et al. Meaningful family relationships: neurocognitive buffers of adolescent risk taking J Cogn Neurosci 2013;25:374-87.

49. McCormick EM, Qu Y, Telzer EH. Adolescent neurodevelopment of cognitive control and risk-taking in negative family contexts. Neuroimage 2016;124:989-96.

50. Lo WS, Wang MP, Leung LT, et al. School-based smoking survey 2012-13: final survey report. Hong Kong: The University of Hong Kong, 2013. 